\title{
Simulación de los cambios de carbono orgánico del suelo en sistema de cultivo con higuerilla por el modelo RothC
}

\author{
Lucila González Molina(1), Esaú del C. Moreno Pérez ${ }^{(2)}$, Laksmi Reddiar Krishnamurty(2), \\ Aurelio Baéz Pérez ${ }^{(3)}$ y Acosta Mireles Miguel ${ }^{(1)}$
}

\begin{abstract}
(1)Instituto Nacional de Investigaciones Forestales, Agrícolas y Pecuarias (Inifap), Campo Experimental Valle de México, Km 13,5 Carretera Los Reyes-Texcoco. CP 56250 Texcoco, Estado de México, México. E-mail: gonzalez.lucila@inifap.gob.mx, acosta.miguel@inifap.gob.mx (2)Universidad Autónoma Chapingo, Km 38,5, Carretera México-Texcoco, CP 56320 Texcoco, Estado de México, México. E-mail: krishnair@yahoo.com.mx ${ }^{(3)}$ Inifap, Campo Experimental Bajío, Km 6,5 Carretera Celaya-San Miguel de Allende. CP 38010 Celaya, Guanajuato, Mexico. E-mail: baez.aurelio@inifap.gob.mx
\end{abstract}

Resumen - El objetivo del presente trabajo fue la simulación de los cambios del carbono orgánico del suelo (COS), por el modelo RothC, en razón del cambio de uso de suelo del sistema tradicional - asociación maíz-calabaza (TMC) - a sistemas con higuerilla (Ricinus communis): multiestratos (MUL), callejones (CALL) y monocultivo de higuerilla (HIG). Las simulaciones del COS se hicieron para las profundidades de suelo 0-20 y 0-40 cm, para el periodo de 1980-2040, considerándose como línea base al sistema TMC. Las tasas de cambio de COS estimadas con el RothC, en ambas profundidades de suelo, estuvieron en 0,5-1,2, $0,4-0,8,0,3-0,5$ y $0,04-0,1 \mathrm{Mg} \mathrm{ha}^{-1}$ de C por año en los sistemas HIG, MUL, CALL y TMC, respectivamente, y fueron consistentes con las reportadas en la literatura. El desempeño del RothC tuvo $89 \%$ de eficiencia (EF), con $\mathrm{R}^{2}=0,9$, lo que muestra que este modelo puede usarse con información temporal del COS escasa, información de la historia de uso de suelo y mediciones de la entrada de residuos vegetales aéreos y subterráneos en el suelo.

Términos de indexación: Ricinus communis, cambio de uso de suelo, plantas biocombustibles, secuestro de carbono.

\section{Simulation of soil organic carbon changes in crop systems with castor bean using the RothC model}

\begin{abstract}
The objective of this work was to simulate, by the RothC model, the changes in soil organic carbon (SOC) caused by changes of land use - from the traditional maize-squash (TMC) association to systems with castor bean (Ricinus communis): multilayer (MUL), alleys (CALL) and monoculture (HIG). SOC simulations were performed for 0-20 and 0-40 cm soil depths, for the period 1980-2040, considering the TMC system as the base line. SOC change rates estimated with RothC, for both soil depths, were 0.5-1.2, 0.4-0.8, 0.3-0.5 and 0.04-0.1 Mg ha ${ }^{-1} \mathrm{C}$ per year in the HIG, MUL, CALL and TMC systems, respectively, and were consistent with those reported in the literature. RothC perfomance had $89 \%$ efficiency $(\mathrm{EF})$ and $\mathrm{R}^{2}=0.9$, which shows that this model can be used with scarce SOC temporal information, information on the history of land use, and with input measurements of aerial and underground plant residues in the soil.
\end{abstract}

Index terms: Ricinus communis, land use change, biofuel plants, carbon sequestration.

\section{Introducción}

Los cambios en el uso del suelo y adopción de mejores prácticas agrícolas tienen el potencial de aumentar el secuestro de $\mathrm{CO}_{2}$ atmosférico (Post \& Kwon, 2000). El secuestro de carbono (C) en el suelo implica la transferencia de $\mathrm{CO}_{2}$ atmosférico por las plantas y su almacenamiento como carbono orgánico del suelo (COS) (Lal, 2004). No obstante, la falta de datos de COS en experimentos de larga duración dificulta la estimación temporal del almacenamiento o pérdida de $\mathrm{C}$ en el suelo (Kaonga \& Coleman, 2008). En el caso de México, de acuerdo con González Molina et al. (2011), estos experimentos son de corta duración ( $<20$ años), por lo que el uso de modelos de simulación del COS es útil en la predicción de los cambios del COS.

El modelo RothC-26.3 de Coleman \& Jenkinson (2005) es uno de los más usados en la simulación de la dinámica del $\mathrm{C}$ en el suelo, debido a la disponibilidad y simplicidad de los datos de entrada para ejecutarlo 
(Coleman et al., 1997; Liu et al., 2009). En México, se ha evaluado el desempeño del RothC en sistemas agrícolas, forestales y praderas, con resultados de $\mathrm{r}$ de 0,81 a 0,96 (González Molina et al., 2011).

Actualmente, la tendencia mundial de la producción de bioenergéticos ha contribuido para el desarrollo de la Red Nacional de Investigación e Innovación en Bioenergéticos en México. Esta red se ha enfocado principalmente en especies biocombustibles como Jatropha curcas L. (piñon manso) y Ricinus communis L. (higuerilla), que no compiten con las biocombustibles cultivadas para la alimentación humana (Rodríguez \& Zamarripa, 2011). Con base en revisión de literatura, en el caso de la higuerilla se encontró que la información referente al secuestro de $\mathrm{C}$ en el suelo y el contenido de $\mathrm{C}$ en su biomasa aérea y subterránea se desconoce. En Oaxaca, la higuerilla se cultivó en diez mil hectáreas, en las décadas de 1980 y 1990, reducidas para 3.600 ha (Arredondo, 2004); en Zaachila, Oaxaca, es tradicionalmente cultivada por los campesinos hace más de 40 años.

El objetivo del presente trabajo fue la simulación de los cambios del carbono orgánico del suelo (COS), por el modelo RothC, en razón del cambio de uso de suelo del sistema tradicional - asociación maíz-calabaza (TMC) - a sistemas con higuerilla (Ricinus communis): multiestratos (MUL), callejones (CALL) y monocultivo de higuerilla (HIG).

\section{Materiales y Métodos}

Este trabajo se realizó entre marzo de 2010 y agosto de 2011, en parcelas experimentales ubicadas en la comunidad de Santa María Roaló, del Municipio de Zaachila, Valles Centrales de Oaxaca, México, y son parte del Proyecto Nacional de Biocombustibles. Los sitios de estudio se localizan a $16^{\circ} 54^{\prime} \mathrm{N}$ y $96^{\circ} 46^{\prime} \mathrm{W}$, a $1.490 \mathrm{~m}$ de altitud. Presentan una precipitación anual promedio de $744 \mathrm{~mm}$ y temperatura media de $20^{\circ} \mathrm{C}$ (Comisión Nacional del Agua, 2011). El clima, según la clasificación de Köppen, modificada por García de Miranda (1986), es $\mathrm{C}_{\mathrm{a}} \mathrm{W}_{\mathrm{o}}$, semicálido del grupo $\mathrm{C}$, el más seco de los subhúmedos, con lluvias en verano; la temperatura del mes más cálido es menor que $22^{\circ} \mathrm{C}$. El suelo es Regosol Eútrico de textura gruesa (Food and Agriculture Organization of the United Nations, 1998). El $\mathrm{pH}$ y el contenido promedio de $\mathrm{COS}\left(\mathrm{g} \mathrm{kg}^{-1}\right), \mathrm{N}$ total $\left(\mathrm{g} \mathrm{kg}^{-1}\right)$, P-Olsen $\left(\mathrm{mg} \mathrm{kg}^{-1}\right)$ y K $\left(\mathrm{mg} \mathrm{kg}^{-1}\right)$, fueron de 6,6 , $1,2,0,05,23$ y 14,4 , respectivamente.

Los sistemas de cultivo fueron los siguientes: tradicional TMC, con los cultivos de maíz (Zea mays L.) intercalados con calabaza (Cucurbita sp.), cultivados con distancia entre plantas de $0,75 \mathrm{~m}$ y $1,5 \mathrm{~m}$, respectivamente; multiestrato (MUL), con los cultivos de higuerilla, calabaza y maíz, y el arreglo de las hileras fue de maíz, calabaza, maíz e higuerilla, y así sucesivamente, con distancia entre plantas de higuerilla de $1,5 \mathrm{~m}$, de maíz de 0,75 y de calabaza 1,5; monocultivo de higuerilla con edad de 24 meses (HIG), cultivada con distancia entre plantas de 1,5 m; y callejones (CALL), con dos hileras de maíz por una de higuerilla, y así sucesivamente, con distancia entre plantas de 1,5 m y $0,75 \mathrm{~m}$, respectivamente. En los cuatro sistemas de cultivo, la distancia entre hileras fue de $0,75 \mathrm{~m}$.

El modelo RothC, de acuerdo con Coleman \& Jenkinson (2005), es un modelo de múltiples compartimentos, y el almacén COS se divide en cuatro compartimentos activos y uno pasivo, por su velocidad de descomposición: material vegetal de fácil descomposición (MVD, que permanece en el suelo por 0,165 años); material vegetal resistente (MVR, 2,71 años); biomasa microbiana (BIO, 1,69 años); materia orgánica humificada (HUM, 49 años); y materia orgánica inerte (MOI, con duración en el suelo de 1.980 años). En la simulación del RothC, los residuos vegetales (RV) que entran al suelo se separan en MVD y MVR, dependiendo del origen (cultivos, pastizales o bosques). Los compartimentos MVD y MVR se descomponen para formar $\mathrm{BIO}, \mathrm{HUM}$ y $\mathrm{CO}_{2}$ en razón del contenido de arcilla. La BIO y el HUM generados se descomponen para producir más BIO y HUM. Los compartimentos activos experimentan descomposición por cinética de primer orden, y cada uno presenta una tasa constante de descomposición $(\mathrm{k})$ por año para MVD (10), MVR $(0,3)$, BIO $(0,66)$, y HUM - con $\left[0,2: \mathrm{Y}=\mathrm{Y}_{0}\left(1-\mathrm{e}^{- \text {abckt }}\right)\right]$, en que: $\mathrm{Y}_{0}$ es el $\mathrm{C}$ inicial; $\mathrm{a}$, $\mathrm{b}$ y c son los factores que modifican a $\mathrm{k}$ (temperatura, humedad y cobertura del suelo, respectivamente); y t es 1/12 para obtener la tasa de descomposición al final de cada mes. La MOI $\left(\mathrm{Mg} \mathrm{ha}^{-1}\right)$ es obtenida de acuerdo con Falloon et al. (1998) por MOI =0,049 $\times \mathrm{COT}^{1,139}$, en que COT es el carbono orgánico $\left(\mathrm{Mg} \mathrm{ha}^{-1}\right)$.

Los parámetros de entrada incluyen las siguientes informaciones: climática - temperatura del aire 
$\left(\mathrm{TPM},{ }^{\circ} \mathrm{C}\right)$, precipitación (PPM, mm) y evaporación (EPM, mm) promedio mensual; edáfica - contenido de arcilla y profundidad de muestreo del suelo; entrada de $\mathrm{C}$ al suelo - entrada mensual de $\mathrm{C}$ de $\mathrm{RV}$ y abonos orgánicos (AO); e información sobre la cobertura vegetal del suelo - para verificar si suelo está desnudo o con cobertura vegetal, la relación MVD/MVR y MOI obtenido de la ecuación de Falloon et al. (1998). La diferencia de los RV que entran al suelo se da por los valores default que se originan de la relación MVD/MVR: un valor de 0,25 para la vegetación forestal (por ejemplo, el 20\% de los restos vegetales es MVD, y el $80 \%$ es MVR); de 1,44 para los cultivos y pastos con manejo (el 59\% es MVD, y el 41\% es MVR); y de 0,67 para los pastos no explotados y matorrales (incluso sabana) (Coleman \& Jenkinson 1996). Con las informaciones climáticas y edáficas, se hace un archivo de clima-suelo, y con la información mensual de RV y $\mathrm{AO}$, y los meses con cobertura vegetal del suelo, se construyen los archivos de manejo de suelo.

La simulación del C con RothC se desarrolló en dos etapas: inicialización y elaboración de escenarios de C. En ambas etapas, se usaron los archivos de clima-suelo y de manejo de suelo correspondientes. La inicialización consistió en obtener el contenido inicial $\mathrm{C}$ de los compartimentos activos, en los sistemas de estudio en la condición de equilibrio. Se asumió que los suelos del presente estudio estuvieron en la condición de equilibrio, pues, de acuerdo con Nieto et al. (2010), los suelos que han permanecido con el mismo uso por más de 30 años, alcanzan esta condición. El estado de equilibrio se asumió considerándose la información de uso de suelo de la cartografía del Inegi, entre marzo de 1979 y marzo de 1980 (Comisión Nacional para el Conocimiento y Uso de la Biodiversidad, 1999) y se corroboró con la de los agricultores del sitio de estudio. Estos indicaron que las parcelas en estudio han sido cultivadas con la asociación maíz-calabaza hace más de 40 años. El RothC se ejecutó iterativamente para 10 mil años atrás, con el valor de $\mathrm{C}$ de la $\mathrm{MOI}$, la relación MVD/MVR y la entrada de $\mathrm{C}$ al suelo de los RV medidos en el sistema TMC. Se elaboraron escenarios de manejo del suelo del periodo entre 1980 y 2040, con base en la información histórica de uso del suelo y de los archivos de manejo del suelo generados para cada sistema. Los escenarios fueron los siguientes: TMC, el sistema se mantuvo sin cambio de 1980-2040; MUL, el sistema TMC se simuló de 1980 a 2004 y cambió a
MUL de 2005 a 2040; HIG, el sistema TMC se simuló de 1980 a 2007 y cambió a HIG de 2008 a 2040; y CALL, el sistema TMC se simuló de 1980 a 2004 y cambió a CALL de 2010 a 2040.

Se obtuvieron los datos de entrada medidos y asumidos por el RothC y las condiciones de simulación en el periodo de 1980 a 2040. Los datos climáticos TPM, PPM y EPM corresponden al periodo 19712000 de la Estación Zimatlán de Álvarez (Comisión Nacional del Agua, 2011) ubicada a $16^{\circ} 52^{\prime} \mathrm{N}, 96^{\circ} 45^{\prime} \mathrm{W}$. El contenido de arcilla, obtenido en el presente estudio, fue de $130 \mathrm{~g} \mathrm{~kg}^{-1}$. El COS se midió en 2011, en los sistemas TMC, HIG y MUL; en el caso del sistema CALL, no se tuvieron mediciones de COS, debido a que se estableció en 2010. El COS fue medido en los incrementos de profundidad del suelo 0-20 y 20-40 cm, y el usado en las simulaciones con el RothC fue el de $0-20 \mathrm{~cm}$ y $0-40 \mathrm{~cm}$. El COS 0-40 fue la suma de los promedios obtenidos en los incrementos $(0-20+20-40 \mathrm{~cm})$. El tiempo con cobertura vegetal del suelo fue de 12 meses, en MUL, HIG y CALL, y de siete meses en TMC. La relación MVD/MVR en los escenarios fue de 1,44 (no se consideró de 0,25 para el escenario de HIG, como lo considera el RothC para especies arbustivas, debido a que esta especie se corta cada dos o tres años para renovar la producción de su semilla). El valor de la MOI fue el promedio obtenido en los puntos de muestreo con el uso de la ecuación de Falloon et al. (1998). Los RV considerados como entrada de $\mathrm{C}$ al suelo, en cada sistema, fueron medidos en los cuatro sistemas; y la distribución de RV $\left(\mathrm{Mg} \mathrm{ha}^{-1} \mathrm{C}\right)$ fue uniforme en los meses con cobertura del suelo, debido a que la forma en que las entradas son distribuidas afectan poco los cálculos de COS (Coleman y Jenkinson, 1996). En el Cuadro 1, se presenta parte de la información necesaria para simular los cambios de COS con RothC, en los sistemas con higuerilla, a profundidades de $0-20$ y $0-40 \mathrm{~cm}$.

La medición directa del COS se llevó a cabo con el método descrito por Monreal et al. (2005). En el año 2011 , en parcelas de aproximadamente $1.000 \mathrm{~m}^{2}$, se tomaron cuatro muestras de suelo en los incrementos de profundidad 0-20 y $20-40 \mathrm{~cm}$, en un diseño experimental completamente al azar. El muestreo se realizó con una barrena de $4,8 \mathrm{~cm}$ de diámetro y 20 de longitud. Las muestras de suelo se secaron al aire, después de registrar su peso; con auxilio de pinzas, se retiraron raíces, restos vegetales y animales visibles al 
ojo desnudo y se los tamizaron a $2 \mathrm{~mm}$. La densidad aparente $(\mathrm{Da})$ fue determinada por la relación entre el peso del suelo seco y el volumen del suelo. El peso de suelo seco se constituyó del suelo mineral más el humificado, y no se consideró el peso de los RV y piedras; el volumen del suelo se cálculó al descontar del volumen total el volumen de los RV y piedras. Para la determinación analítica del $\mathrm{C}$, la concentración de COS de una submuestra se midió por combustión seca, en un analizador automático de C, Shimadzu TOC 5000. El COS $\left(\mathrm{Mg} \mathrm{ha}^{-1}\right)$ se calculó de acuerdo con el enfoque de Ellert \& Bettany (1995), que elimina el efecto de las diferencias en la masa del suelo, cuando se comparan las cantidades de $\mathrm{C}$ en suelos con diferente uso, lo que se logra con el uso de una masa equivalente de suelo (MES), conforme la ecuación de Ellert \& Bettany $(1995)-\mathrm{T}_{\text {add }}=\left(\mathrm{M}_{\text {suelo, equiv }}-\mathrm{M}_{\text {suelo, superficial }}\right) / \boldsymbol{\ell} \mathrm{b}-$, en que: $\mathrm{T}_{\text {add }}$ es el espesor adicional de la capa subsuperficial, necesario para obtener la MES (m); $\mathrm{M}_{\text {suelo, equiv }}=\mathrm{MES}$, masa de suelo con mayor peso $\left(\mathrm{Mg} \mathrm{ha}^{-1}\right) ; \mathrm{M}_{\text {suelo, superficial }}$ es la masa de suelo en la capa superficial u horizonte genético $\left(\mathrm{Mg} \mathrm{ha}^{-1}\right), \boldsymbol{\ell} \mathrm{b}$ es la densidad aparente de la capa subsuperficial $\left(\mathrm{Mg} \mathrm{m}^{-3}\right) ; \mathrm{y}_{\text {carbono, equiv }}=\mathrm{M}_{\text {carbono, }}$ superficial $+\mathrm{M}_{\text {carbono, Tadd, en que }} \mathrm{M}_{\text {carbono, equiv }}$ es la masa de $\mathrm{C}$ por unidad de área en una MSE $\left(\mathrm{Mg} \mathrm{ha}^{-1}\right), \mathrm{M}_{\text {carbono, }}$ superficial es la la masa de $\mathrm{C}$ en la capa superficial del suelo

Cuadro 1. Información general para simular los cambios de COS (1980-2040) con RothC, en los sistemas con higuerilla, para las profundidades de 0-20 y 0-40 cm, en Santa María Roalo, Oaxaca, Mexico.

\begin{tabular}{|c|c|c|c|c|}
\hline \multirow[t]{2}{*}{ Sistema } & $\mathrm{COS}_{\text {medido }}$ & MOI & $\mathrm{RV}_{\mathrm{s}_{\text {medidos }}}$ & \multirow{2}{*}{$\begin{array}{c}\text { CVS } \\
\text { (Meses) }\end{array}$} \\
\hline & \multicolumn{3}{|c|}{----------(Mg ha-1 por año)---------- } & \\
\hline & \multicolumn{4}{|c|}{ Profundidad 0-20 cm } \\
\hline $\mathrm{TMC}_{\text {equilibrio }}$ & 13 & 1,37 & 2,1 & 7 \\
\hline $\mathrm{TMC}$ & 18 & 1,37 & 2,1 & 7 \\
\hline MUL & 20 & 1,37 & 3,9 & 12 \\
\hline HIG & 21 & 1,37 & 5,0 & 12 \\
\hline \multirow[t]{2}{*}{ CALL } & - & 1,37 & 3,4 & 12 \\
\hline & \multicolumn{4}{|c|}{ Profundidad $0-40 \mathrm{~cm}$} \\
\hline $\mathrm{TMC}_{\text {equilibrio }}$ & 32 & 3,43 & $4,7^{(1)}$ & 7 \\
\hline $\mathrm{TMC}$ & 38 & 3,43 & 2,1 & 7 \\
\hline MUL & 44 & 3,43 & 3,9 & 12 \\
\hline HIG & 45 & 3,43 & 5,0 & 12 \\
\hline CALL & - & 3,43 & & 12 \\
\hline
\end{tabular}

${ }^{(1)}$ Obtenido en la simulación inversa con el modelo RothC. TMC, sistema tradicional de asociación maíz-calabaza; MUL, multiestratos; HIG, monocultivo de higuerilla; y CALL, callejones. RV, residuos vegetales; MOI, materia orgánica inerte; COS, carbono orgánico del suelo; CVS, cobertura vegetal del suelo.
$\left(\mathrm{Mg} \mathrm{ha}^{-1}\right), \mathrm{y}_{\text {carbono, Tadd }}$ es la masa de $\mathrm{C}$ adicional en la capa subsuperficial del suelo $\left(\mathrm{Mg} \mathrm{ha}^{-1}\right)$.

Se estimó la cantidad de $\mathrm{C}$ aportado por las biomasas aérea y subterránea de los cultivos de maíz, calabaza e higuerilla, que integran los sistemas de estudio. En el caso del cultivo de higuerilla, debido a que no se encontraron reportes en la literatura, se procedió a hacer las mediciones de $\mathrm{C}$ en sus componentes estructurales con dos plantas de higuerilla, con tiempo de establecimiento de 6 y 24 meses, de los sistemas MULy HIG, respectivamente. La planta fue seccionada en las estructuras de raíz, tallo, peciolo, ramas, hoja e inflorescencia (la planta de seis meses no presentó inflorescencia). En el caso de las raíces, se extrajo la mayor cantidad posible con auxilio de herramientas de excavación (pala, pico, barreta), para la raíz principal, hasta la profundidad de $0,57 \mathrm{~m}$ (MUL) y de $0,70 \mathrm{~m}$ (HIG) y, para las raíces secundarias, en ambos sistemas, en promedio en un metro de diámetro. En campo, se obtuvo de cada una de estas estructuras el peso fresco total y se tomó una muestra representativa. Las muestras representativas se colocaron en una estufa a $75^{\circ} \mathrm{C}$, durante 48 horas, para determinar el contenido de humedad y, posteriormente, se calculó la materia seca, por la multiplicación del contenido de humedad por el peso fresco de las estructuras. Para la determinación analítica del $\mathrm{C}$ de las estructuras vegetales, se tomaron muestras representativas de $100 \mathrm{~g}$ y se las molieron en un molino provisto de malla de $1 \mathrm{~mm}$. Se obtuvo una submuestra de $5 \mathrm{~g}$, para determinar la concentración de $\mathrm{C}$ en un analizador automático de $\mathrm{C}$, Shimadzu, TOC 5000 .

Se midió la cantidad de $\mathrm{C}$ - proveniente de los $\mathrm{RV}$ aéreos y subterráneos - aportada al suelo. Los RV de la superficie del suelo, herbáceos principalmente, fueron colectados dentro de un aro con diámetro de $52 \mathrm{~cm}$. La entrada total de RV $\left(\mathrm{Mg} \mathrm{ha}^{-1}\right)$ fue el promedio de tres muestreos, en forma aleatoria, y en dos ocasiones: la segunda semana de noviembre, durante la cosecha de maíz o calabaza, según el sistema que correspondía; y la segunda semana de febrero, durante corte de rastrojo de maíz. En el cultivo de maíz, los RV aéreos y subterráneos se determinaron a partir de la biomasa aérea, considerándose un índice de cosecha de 0,4 y un rendimiento de grano $2,5 \mathrm{Mg} \mathrm{ha}^{-1}$, con base en los estudios de Ruíz Vega (1998) y de la Oficina Estatal de Información para el Desarrollo Rural Sustentable (2005). Las raíces se estimaron como $15 \%$ de la BA, 
y los restos de la cosecha anterior fueron $18 \%$ de la BA. La proporción de $15 \%$ fue tomada del estudio de Kuzyakov \& Domanski (2000), quienes indican que, en cereales, 10 a $15 \%$ del $\mathrm{C}$ total capturado por el proceso de fotosíntesis es almacenado en este órgano; la proporción de $18 \%$ se consideró del estudio de Pérez Olvera et al. (2000), quienes estudiaron el aporte de RV al suelo en maíz después de la cosecha. En higuerilla, los RV que entraron al suelo fueron la raíz y la hojarasca, medidas en plantas con tiempo de establecimiento de 6 y 24 meses. En el caso del cultivo de calabaza y otras hierbas, se consideró que, en los RV aéreos del cultivo de calabaza, hierbas estuvieron incluidas en lo cuantificado dentro del aro. Los RV subterráneos se calcularon de las raíces que se retiraron de las muestras de suelo.

Para evaluación del desempeño del modelo RothC, en la simulación de los cambios de COS, se usaron los siguientes criterios: coeficiente de determinación $\left(\mathrm{R}^{2}\right)$; raíz del cuadrado medio del error (RMSE) (\%); y la eficiencia del modelo (EF) expresada por Smith et al. (1997). Se compararon los valores de COS promedio medidos en 2011 y los obtenidos en la simulación con RothC, para las profundidades $0-20$ y $0-40 \mathrm{~cm}$, con una prueba de t para datos pareados a $5 \%$ de probabilidad.

\section{Resultados y Discusión}

El COS medido en los sistemas con higuerilla, para las profundidades de suelo 0-20, 20-40 y 0-40 cm, se presenta en el Cuadro 2. El sistema HIG tuvo los valores más altos de COS, seguido de los sistemas MUL y TMC, y la misma tendencia se encontró con respecto a la entrada de RV medidos al suelo (Cuadro 3). Estos resultados fueron consistentes con los de Powlson et al. (2011), quienes, al comparar el efecto de adición y no adición de rastrojo en 23 experimentos de larga duración, encontraron que la tendencia general fue un incremento en las concentraciones de $\mathrm{C}$ del suelo por la incorporación de residuos vegetales.

En el cultivo de maíz, la concentración de $\mathrm{C}$ en la biomasa fue la reportada en el estudio de Acosta Mireles (2003), ubicado en la Sierra Norte de Oaxaca, en que los valores de este elemento no variaron, y el promedio fue de $47 \%$. El C aportado por el cultivo de calabaza que se incluyó en los muestreos de hierbas, por la consistencia de esta planta, tuvo un valor de $45 \%$. En el caso de higuerilla, se encontró una concentración promedio de $\mathrm{C}$ en las estructuras de $41 \%$ (raíz, 41\%; tallo, $42 \%$; hoja, $43 \%$; peciolo, $39 \%$; rama, $40 \%$; e inflorescencia, $41 \%$ ), resultados que son similares a los del estudio de Figueroa Navarro (2001) sobre diferentes especies arbóreas en Oaxaca. El aporte de RV de los cultivos que integran los sistemas de estudio, en las simulaciones del COS para las profundidades 0-20 y 0-40 cm, se presenta en el Cuadro 3 .

El COS simulado con el RothC, después del cambio de uso del TMC a los sistemas con higuerilla entre 1980 y 2040 (Figura 1 y Cuadro 4), tuvo el siguiente orden: $\mathrm{HIG}>\mathrm{MUL}>\mathrm{CALL}>\mathrm{TMC}$, resultados consistentes con la tendencia general encontrada por Nair et al. (2009): forestales>agroforestales> plantaciones de árboles>cultivos; sin embargo, según estos investigadores, la estimación de $\mathrm{C}$ almacenado varía en gran medida, en consecuencia del tipo de manejo del sistema, la cantidad y calidad de la entrada de RV al suelo provenientes de los componentes arbóreos y no arbóreos y, también, de las propiedades del suelo como la estructura y su agregación.

Cuadro 2. Carbono orgánico del suelo ( $\mathrm{COS},\left(\mathrm{Mg} \mathrm{ha}^{-1}\right)$ medido en los sistemas de estudio en las profundidades de suelo de 0-20, 20-40 y 0-40 cm.

\begin{tabular}{|c|c|c|c|c|c|}
\hline \multirow[t]{2}{*}{ Sistema $^{(1)}$} & \multicolumn{2}{|c|}{$0-20 \mathrm{~cm}$} & \multicolumn{2}{|c|}{$20-40 \mathrm{~cm}$} & \multirow{2}{*}{$\frac{0-40 \mathrm{~cm}}{\operatorname{Media}^{(2)}}$} \\
\hline & Media $^{(2)}$ & DE & Media & $\mathrm{DE}$ & \\
\hline TMC & 18 & 6 & 20 & 7 & 38 \\
\hline MUL & 23 & 3 & 21 & 5 & 44 \\
\hline HIG & 24 & 5 & 21 & 6 & 45 \\
\hline
\end{tabular}

${ }^{(1)} \mathrm{TMC}$, sistema tradicional de la asociación maíz-calabaza; MUL, multiestratos; HIG, monocultivo de higuerilla; y CALL, callejones.

${ }^{(2)} \mathrm{COS}$ usado en la simulación con RothC $\left(\mathrm{COS}_{\mathrm{O}-40}=\mathrm{COS}_{0-20 \mathrm{~cm}}+\mathrm{COS}\right.$ promedio $\left._{20-40 \mathrm{~cm}}\right)$. DE, desviación estándar.

Cuadro 3. Aporte carbono ( $\left.\mathrm{C}, \mathrm{Mg} \mathrm{ha}^{-1}\right)$ de residuos vegetales de los cultivos que integran los sistemas de estudio, usados en la simulación los cambios de COS (1980-2040) con RothC, para las profundidades de $0-20$ y $0-40 \mathrm{~cm}$.

\begin{tabular}{|c|c|c|c|c|c|c|}
\hline \multirow[t]{2}{*}{ Sistema $^{(\mathbf{l})}$} & \multicolumn{2}{|c|}{ Maíz } & \multirow{2}{*}{$\begin{array}{c}\text { Higuerilla } \\
\text { Raíz }\end{array}$} & \multicolumn{2}{|c|}{ Cultivos+herbáceas } & \multirow[t]{2}{*}{ Total } \\
\hline & Raíz & Aéreo & & Raíz & Aéreo & \\
\hline TMC & 0,42 & 0,51 & & 0,01 & 1,20 & 2,10 \\
\hline MUL & 0,42 & 0,51 & 0,21 & 0,05 & 2,80 & 3,98 \\
\hline HIG & & & 1,00 & 0,01 & 4,00 & 5,01 \\
\hline CALL & 0,42 & 0,51 & 0,21 & 0,01 & 2,30 & 3,40 \\
\hline
\end{tabular}

${ }^{(1)} \mathrm{TMC}$, sistema tradicional de la asociación maíz-calabaza; MUL, multiestratos; HIG, monocultivo de higuerilla; y CALL, callejones. 
En el sistema TMC, los cambios de C son constantes e imperceptibles; en contraste, los otros sistemas muestran un desequilibrio, ocasionado por el cambio de uso de suelo y entrada de RV al suelo y, posteriormente, una tendencia al estado de equilibrio. En el estado de equilibrio, las entradas de $\mathrm{C}$ al suelo son similares a las salidas en forma de $\mathrm{CO}_{2}$ atmosférico (Fearnside \& Barbosa, 1998). Según Nieto et al. (2010), los suelos pueden alcanzar un estado de equilibrio, si el manejo del suelo se mantiene por un periodo superior a 30 años. Johnson et al. (1995) indicaron que el COS alcanza un nuevo equilibrio, algún tiempo después de la adopción de la nueva práctica de manejo. Por su parte, Hutchinson et al. (2007) mostraron que si el manejo agrícola promueve secuestro de $\mathrm{C}$, este continuará hasta un estado de equilibrio asintótico, que durará mientras el manejo no mude y las condiciones climáticas se mantengan similares.

Elorden en que pueden alcanzar el estado de equilibrio en el suelo, como se observa en la Figura 1, fue inverso

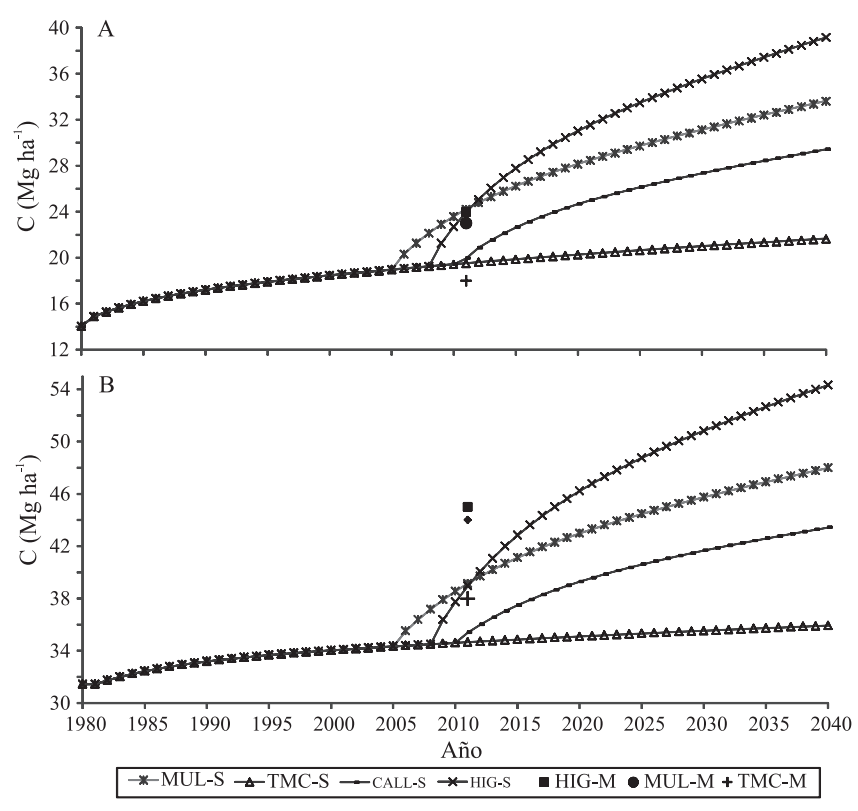

Figura 1. Carbono orgánico del suelo (COS) simulado con el RothC, en los sistemas: monocultivo de higuerilla multiestratos, callejones y tradicional maíz-calabaza, en un periodo de 60 años, en Santa María Roalo, Oaxaca, Mexico. A, simulación de $\mathrm{COS}$ para la profundidad 0-20 cm; B, simulación de COS para la profundidad 0-40 $\mathrm{cm}$. TMC, sistema tradicional de la asociación maíz-calabaza; MUL, multiestratos; HIG, monocultivo de higuerilla; y CALL, callejones. $-\mathrm{M}$, COS medido, $\mathrm{y}-\mathrm{S}=\mathrm{COS}$ simulado. al C secuestrado: TMC $>$ CALL $>$ MUL $>$ HIG. Esto puede explicarse por el tiempo de conversión o cambio del COS que, según Jenkinson \& Rayner (1977), se define como el movimiento del C orgánico (migración), a través de un volumen dado de suelo, y es la relación entre el COS y la entrada anual de RV. El tiempo de conversión en el sistema TMC fue de 9 y 18 años, en MUL de 6 y 11 años y en HIG de 5 y 9 años, para las profundidades de $0-20$ y $0-40 \mathrm{~cm}$, respectivamente. Cuando se compara el tiempo de conversión con la profundidad, se encuentra que los valores más altos de conversión indican la estabilización del C en el suelo, como es el caso del sistema TMC, y que los más bajos son una migración más rápida del $\mathrm{C} \mathrm{y}$, por lo tanto, indican que el suelo está más lejano del estado de equilibrio, caso de los sistemas HIG y MUL (Figura 1, Cuadro 4).

La simulación de los escenarios de cambio de uso de suelo, en los sistemas con higuerilla, indicaron cambios de COS en la profundidad $0-20 \mathrm{~cm}$, en general menores que los de la profundidad 0-40 cm, lo que puede explicarse porque el modelo considera factores como la humedad y la temperatura, que afectan las tasas de descomposición correspondientes a los compartimentos activos del COS. En la profundidad de 0-20 cm, las tasas de cambio ( $\mathrm{Mg} \mathrm{ha}^{-1}$ por año) fueron del orden de 0,08 a 1,2 , y a la profundidad de $0-40 \mathrm{~cm}$ fueron de 0,04 a 1,1. Las tasas de cambio por sistema estuvo en los rangos de $0,5-1,2,0,4-0,8,0,3-0,5$ y 0,04-0,1 en HIG, MUL, CALLy TMC, respectivamente (Cuadro 4). Estos resultados están de acuerdo con la escala mundial de 0,02 a 0,76 de la tasa de secuestro

Cuadro 4. Cambios de carbono orgánico del suelo (COS) simulados con RothC (1980-2040), después de 5, 15 y 40 años en sistemas con higuerilla, para las profundidades de 0-20 y $0-40 \mathrm{~cm}$.

\begin{tabular}{lcccc}
\hline $\begin{array}{l}\text { Cambio de uso } \\
\text { de suelo (años) }\end{array}$ & TMC & $\begin{array}{c}\text { MUL } \\
\text {--ord }\end{array}$ & HIG & CALL \\
\hline 5 & 0,07 & 0,79 & 1,16 & 0,54 \\
15 & 0,08 & 0,59 & 0,85 & 0,43 \\
30 & 0,08 & 0,44 & 0,51 & 0,33 \\
\hline \multicolumn{5}{c}{ Profundidad 0-20 cm } \\
5 & 0,04 & 0,71 & 1,11 & 0,49 \\
15 & 0,05 & 0,55 & 0,85 & 0,38 \\
30 & 0,05 & 0,41 & 0,52 & 0,29 \\
\hline
\end{tabular}

TMC, sistema tradicional de la asociación maíz-calabaza; MUL, multiestratos; HIG, monocultivo de higuerilla; y CALL, callejones. 
(Mg C ha-1 por año), reportada por Lal (2001) para los cultivos que adoptan sistemas de manejo mejorado, y de 0,1 a 1,3 en el caso de los cultivos que cambian para manejo de suelo con cero labranza.

En general, el modelo RothC presentó un buen desempeño en los escenarios de simulación de los sistemas con higuerilla, con un valor de EF de $89 \%$, de $\mathrm{R}^{2}$ de 0,9 y de la ordenada al origen de la ecuación de regresión lineal simple de 0,92 (Figura 2). El valor 11 de RMSE fue inferior a los obtenidos en los estudios de González et al. (2010) y González Molina et al. (2011), donde se presentó información del desempeño del RothC en sistemas agrícolas de México; en el primero, los sistemas evaluados se agruparon en sistemas con y sin entrada de RV, y en el segundo, los sistemas se evaluaron en condiciones de ladera, con valor de RMSE de18. En otras partes del mundo y en sistemas agrícolas, forestales y praderas, el RothC se ha usado para predecir los cambios del COS (Coleman et al., 1997; Falloon et al., 1998; Falloon \& Smith, 2002).

De acuerdo con los resultados de la prueba de t, no hubo diferencias estadísticas significativas entre los pares de valores de COS medidos ( $32 \pm 12$ ) y simulados

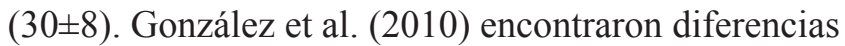
significativas entre COS medido y simulado, en razón principalmente de la variabilidad espacial reportada; $\sin$ embargo, en el presente estudio, las simulaciones con el RothC mostraron las tendencias de los cambios del COS con un valor de $\mathrm{R}^{2}$ de 0,76 y una EF del modelo de 0,6. Según el IPCC (2003), es más válido indicar

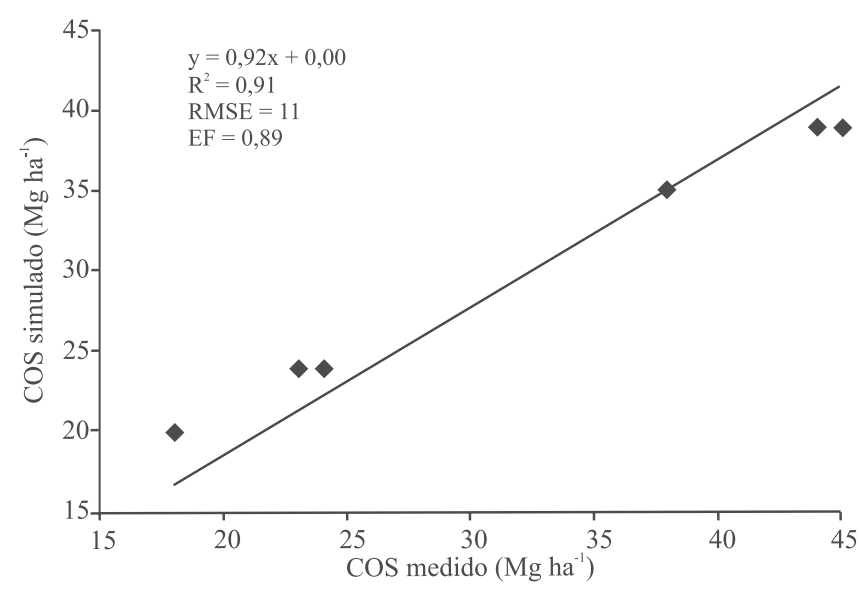

Figura 2. Comparación de carbono orgánico del suelo (COS) promedio contra COS promedio simulado con el RothC, en diferentes sistemas, para las profundidades de suelo de 0-20 y $0-40 \mathrm{~cm}$. las tendencias del COS en el tiempo do que reportar valores absolutos de los cambios de COS, a pesar de que la incertidumbre reportada en los métodos usados en el primer caso sea mayor.

\section{Conclusiones}

1. Es posible estimar las tasas de secuestro de COS de los sistemas con higuerilla, consistentes con las reportadas en otros trabajos.

2. El RothC tuvo un desempeño favorable, mediante la simulación de escenarios de cambios de uso de suelo, alimentado con escasa información temporal de COS, considerándose la información de la historia de uso del suelo y la información de mediciones de la entrada al suelo de residuos vegetales aéreos y subterráneos al suelo de los cultivos que integraron los sistemas de estudio.

\section{Referencias}

ACOSTA MIRELES, M. Diseño y aplicación de un método para medir los almacenes de carbono en sistemas con vegetación forestal y agrícolas de ladera en México. 2003. 89p. Tesis (Doctor) - Programa Forestal Colegio de Postgraduados, Montecillo.

ARREDONDO, V.C. Evaluación de componentes tecnológicos para la producción de higuerilla Ricinus communis L. en Valles Centrales de Oaxaca. Naturaleza y Desarrollo, v.2, p.5-16, 2004.

COLEMAN, K.; JENKINSON, D.S. RothC-26.3 - a model for the turnover of carbon in soil. In: POWLSON, D.S.; SMITH, P.; SMITH, J.U. (Ed.). Evaluation of soil organic matter models: using existing long-term datasets. Berlin: Springer, 1996. p.237-246.

COLEMAN, K.; JENKINSON, D.S. ROTHC-26.3 - a model for the turnover of carbon in soil: model description and windows users guide. Harpenden: Rothamsted Research, 2005. 43p.

COLEMAN, K.; JENKINSON, D.S.; CROCKER, G.J.; GRACE, P.R.; KLIR, J.; KORSCHENS, M.; POULTON, P.R.; RICHTER, D.D. Simulating trends in soil organic carbon in long-term experiments using RothC-26.3. Geoderma, v.81, p.29-44, 1997.

COMISIÓN NACIONAL DEL AGUA. Servicio Meteorológico Nacional. Normales climatológicas 1971-2000: estación: 00020209, Z. de Alvarez. 2011. Disponible en: <http://smn.cna. gob.mx/climatologia/normales/estacion/oax/NORMAL20209. TXT>. Acceso en: 4 enero 2011.

COMISIÓN NACIONAL PARA EL CONOCIMIENTO Y USO DE LA BIODIVERSIDAD. Uso de suelo y vegetación modificado por Conabio. México: Conabio, 1999. 1 mapa. Escala: 1:1000000.

ELLERT, B.H.; BETTANY, J.R. Calculation of organic matter and nutrients stored in soils under contrasting on management regimes. Canadian Journal of Soil Science, v.75, p.529-538, 1995. 
FALLOON, P.; SMITH, P. Simulating SOC changes in long-term experiments with RothC and CENTURY: model evaluation for a regional scale application. Soil Use and Management, v.18, p.101-111, 2002.

FALLOON, P.; SMITH, P.; COLEMAN, K.; MARSHALL, S. Estimating the size of the inert organic matter pool from total soil organic carbon content for use in the Rothamsted carbon model. Soil Biology Biochemistry, v.30, p.1207-1211, 1998.

FEARNSIDE, P.M.; BARBOSA, R.I. Soil carbon changes from conversion of forest to pasture in Brazilian Amazonia. Forest Ecology and Management, v.108, p.147-166, 1998.

FIGUEROA NAVARRO, C.M. Comparación de la concentración de carbono en diferentes tipos de vegetación en la Sierra Norte de Oaxaca. 2001. 101p. Tesis (Maestría) - Universidad Autónoma Chapingo, Chapingo.

FOOD AND AGRICULTURE ORGANIZATION OF THE UNITED NATIONS. World reference base for soil resources. Rome: FAO, 1998. (FAO. World soil resources report, 84).

GARCÍA DE MIRANDA, E. Apuntes de climatología. México: UNAM, 1986. 155p.

GONZÁLEZ, L.; ETCHEVERS, J.D.; GONZÁLEZ, J.M.; PAZ, F. Soil organic carbon changes at the plot level in hillside systems. Agriculture, Ecosystems and Environment, v.139, p.508-515, 2010 .

GONZÁLEZ-MOLINA， L.; ETCHEVERS-BARRA， J.D.; PAZ-PELATT, F.; DÍAZ-SOLIZ, H.; FUENTES-PONTES, M.H.; COVALEDA-OCÓN, S.; PANDO-MORENO, M. Performance of the RothC-26.3 model in short-term experiments in Mexican sites and systems. The Journal of Agricultural Science, v.149, p.415-425, 2011.

JENKINSON, D.S.; RAYNER, J.H. The turnover of soil organic matter in some of the Rothamsted classical experiments. Soil Science, v.123, p.298-305, 1977.

JOHNSON, M.G.; LEVINE, E.R.; KERN, J.S. Soil organic matter: distribution, genesis, and management to reduce greenhouse gas emissions. Water Air and Soil Pollution, v.82, p.593-615, 1995.

HUTCHINSON, J.J.; CAMPBELL, C.A.; DESJARDINS, R.L. Some perspectives on carbon sequestration in agriculture. Agricultural and Forest Meteorology, v.142, p.288-302, 2007.

KAONGA, M.L.; COLEMAN, K. Modelling soil organic carbon turnover in improved fallows in Eastern Zambia using the RothC-26.3 model. Forest Ecology and Management, v.256, p.1160-1166, 2008.

KUZYAKOV, Y.; DOMANSKI, G. Carbon input by plants into the soil: review. Journal of Plant Nutrition and Soil Science, v.163, p.421-431, 2000.

LAL, R. Soil carbon sequestration to mitigate climate change. Geoderma, v.123, p.1-22, 2004.
LAL, R. World cropland soils as a source or sink for atmospheric carbon. Advances in Agronomy, v.71, p.145-191, 2001.

LIU, D.L.; CHAN, K.Y.; CONYERS, M.K. Simulation of soil organic carbon under different tillage and stubble management practices using the Rothamsted carbon model. Soil and Tillage Research, v.104, p.65-73, 2009.

MONREAL, C.M.; ETCHEVERS, J.D.; ACOSTA, M.; HIDALGO, C.; PADILlA, J.; LÓPEZ, R.M.; JIMÉNEZ, L.; VELÁQUEZ, A. A method for measuring above- and below-ground $\mathrm{C}$ stocks in hillside landscapes. Canadian Journal of Soil Science, v.85, p.523-530, 2005.

NAIR, P.K.R.; KUMAR, B.M.; NAIR, V.D. Agroforestry as a strategy for carbon sequestration. Journal of Plant Nutrition and Soil Science, v.172, p.10-23, 2009.

NIETO, O.M.; CASTRO, J.; FERNÁNDEZ, E.; SMITH, P. Simulation of soil organic carbon stocks in a Mediterranean olive grove under different soil-management systems using the RothC model. Soil Use and Management, v.26, p.118-125, 2010.

OFICINA ESTATAL DE INFORMACIÓN PARA EL DESARROLLO RURAL SUSTENTABLE. Tarjeta distrital de información estadística básica: Distrito 17 Zaachila. 2005. Disponible en: <http:/www.oeidrus-oaxaca.gob.mx/fichas/tomoII/ distrito17.pdf>. Acceso en: 11 feb. 2011.

POWLSON, D.S.; GLENDINING, M.J.; COLEMAN, K.; WHITMORE, A.P. Implications for soil properties of removing cereal straw: results from long-term studies. Agronomy Journal, v.103, p.279-289, 2011.

POST, W.M.; KWON, K.C. Soil carbon sequestration and land-use change: processes and potential. Global Change Biology, v.6, p.317-327, 2000 .

PÉREZ OLVERA, M.A.; ETCHEVERS BARRA, J.D.; NAVARRO GARZA, H.; NUÑEZ ESCOBAR, R. Aporte de los residuos del cultivo anterior al reservorio de nitrógeno en tepetates. Agrociencia, v.34, p.115-125, 2000.

RODRÍGUEZ, H.R.; ZAMARRIPA, C.A. Competitividad de la higuerilla Ricinus communis para biocombustible en relación a los cultivos actuales en Oaxaca, México. In: REUNIÓN NACIONAL DE INNOVACIÓN AGRÍCOLA, 6., 2011, León. Memoria. México, DF: Instituto Nacional de Investigaciones Forestales, Agrícolas y Pecuarias, 2011. p.353.

RUÍZ VEGA, V. Zonificación agroecológica del maíz de temporal en los Valles Centrales de Oaxaca. I. Determinación del potencial productivo. Terra Latinoamericana, v.16, p.269-275, 1998.

SMITH, P.; SMITH, J.U.; POWLSON, D.S.; MCGILL, W.B.; ARAH, J.R.M.; CHERTOV, O.G. A comparison of the performance of nine soil organic matter models using datasets from seven long-term experiments. Geoderma, v.81, p.153-225, 1997.

Recibido en 4 de mayo de 2012 y aprobado en 8 de octubre de 2012 